\title{
ANOMALOUSLY LARGE TRANSIENT FIELD THROUGH POLARIZED ELECTRON CAPTURE
}

\author{
J.L. EBERHARDT, G. Van MIDDELKOOP, R.E. HORSTMAN and H.A. DOUBT \\ Fysisch Laboratorium, Rijksuniversiteit, Utrecht, The Netherlands
}

Received 4 April 1975

\begin{abstract}
The measured precession for ${ }^{28} \mathrm{Si}\left(2_{1}^{+}\right)$recoiling into magnetized $\mathrm{Fe}$ shows an anomalous increase with initial recoil velocity. This is explained quantitatively by capture of polarized Fe electrons into $2 \mathrm{~s}$ vacancies in the moving ion.
\end{abstract}

Transient magnetic fields have been used for the determination of $\boldsymbol{g}$-factors of short-lived excited states of nuclei recoiling into ferromagnets [1-3]. In these measurements the magnitude of the transient field was estimated by adjusting the Lindhard and Winther (LW) theory [4], which treats the scattering of (polarized) electrons by an ion on slowing-down in a polarized ferromagnet. For realistic screening and the known polarized electron velocity $v_{\mathrm{p}}$ in the ferromagnet (but ignoring possible atomic effects) the theory yields fields, which are a factor of two lower than found experimentally [4], although the dependence on the charge $Z$ of the nucleus on recoil into $\mathrm{Fe}$ is well reproduced.

Most experiments involving the transient field were carried out at relatively low recoil velocities $(v / c<$ $1.6 \%$ ). The velocity dependence of the transient field in $\mathrm{Fe}$ has been tested in three experiments. For ${ }^{196} \mathrm{Pt}$ [5] and ${ }^{103} \mathrm{Rh}$ [6] barely significant deviations from the adjusted LW theory were observed at a recoil velocity $v / c=1.8 \%$. For ${ }^{56} \mathrm{Fe}[7]$, however, large anomalies were found at $v / c=3 \%$ but the deviation was attributed to radiation damage effects.

Several recent experiments $[8,9]$ on light nuclei $(A=18)$ at higher recoil velocities yielded integral precessions twice as large as expected from the adjusted LW theory (ALW) but the nuclear lifetimes were too long (3-7 ps) to exclude the possibility of (large) static hyperfine fields explaining the deviation.

The possibility of an anomalous transient field was investigated in the present work for ${ }^{28} \mathrm{Si}$ recoiling into polarized $\mathrm{Fe}$ at three initial velocities. The recoils were produced by inelastic proton and $\alpha$-particle scattering at the bombarding energies shown in ta-
Table 1

Summary of measured integral precession angles $\phi$ for ${ }^{28} \mathrm{Si}\left(2_{1}^{+}\right)$.

\begin{tabular}{lllll}
\hline Reaction & $\begin{array}{l}E \\
(\mathrm{MeV})\end{array}$ & $\begin{array}{l}\left.E_{\mathrm{i}}{ }^{28} \mathrm{Si}\right) \\
(\mathrm{MeV})\end{array}$ & $\begin{array}{l}v / c \\
(\%)\end{array}$ & $\begin{array}{l}\phi \\
(\mathrm{mrad})\end{array}$ \\
\hline$\left(\mathrm{p}, \mathrm{p}_{1}\right)$ & 4.87 & 0.5 & 0.6 & $0.9 \pm 0.6$ \\
$\left(\alpha, \alpha_{1}\right)$ & 7.50 & 2.8 & 1.5 & $1.42 \pm 0.15$ \\
$\left(\alpha, \alpha_{1}\right)$ & 19.50 & 8.1 & 2.5 & $3.2 \pm 0.5$ \\
\hline
\end{tabular}

ble 1 . The first-excited $2^{+}$state of ${ }^{28} \mathrm{Si}$ is well suited for an integral precession measurement, since its mean life $\tau_{\mathrm{m}}=0.68 \mathrm{ps}$ is comparable to the slowing-down time in $\mathrm{Fe}$, such that static field effects are negligible. The experimental set-up is described in detail in earlier papers $[2,3]$; the latter also reports the ${ }^{28} \mathrm{Si}$ measurement at the intermediate recoil energy. Each experimental point, including the measurement of beam-bending corrections [2] required one week of accelerator time. The measurements clearly show a precession increasing with recoil energy (table 1). The $g$-factor of the state was taken to be 0.54 . Four nuclear model calculations [10-12] yield this value to within $2 \%$. It has been experimentally verified recently [13] for ${ }^{20} \mathrm{Ne}$ and ${ }^{24} \mathrm{Mg}$ that $\mathrm{g}$-factor calculations for even-even $T=0$ nuclei reliably yield values close to $g=0.50$. The measured integral precession angles divided by the $g$-factor are plotted versus the initial recoil velocity in fig. 1 together with the precessions calculated from the LW theory with screening included (full curve; $v_{\mathrm{p}} / v_{\mathrm{o}}=0.78$ with $v_{\mathrm{o}}=c / 137$ ) and from the ALW theory (dashed curve; $v_{\mathrm{p}} / v_{\mathrm{o}}=0.43$ ). The high-velocity point deviates by four standard deviations and by a factor of three from the $\mathrm{LW}$ curve. 


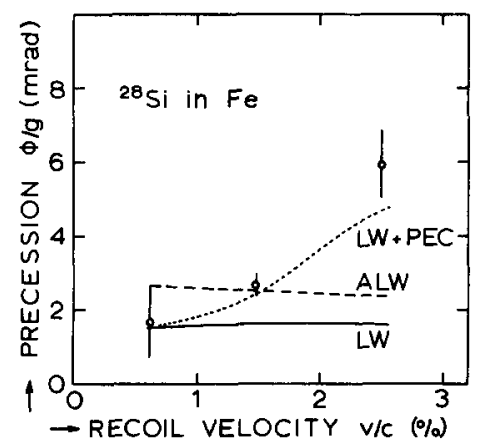

Fig. 1. Measured average precession divided by the $g$-factor as a function of initial recoil velocity for ${ }^{28} \mathrm{Si}$ recoiling in $\mathrm{Fe}$. The Lindhard-Winther (LW) predictions $\left(v_{\mathrm{p}} / v_{\mathrm{o}}=0.78\right.$; screening included; full curve), the adjusted ( $A L W$ ) predictions $\left(v_{\mathrm{p}} / v_{\mathrm{o}}=0.43\right.$; dashed curve) and the sum of the precessions caused by polarized electron scattering (LW) and by polarized electron capture (PEC), dotted curve, are also shown.

The magnitude of the measured effect at high velocity can be accounted for by collisions producing $2 s$ vacancies in the moving ion and the subsequent capture of polarized electrons into these vacancies. The surplus of electrons in Fe with spins along the external field implies more probable capture of electrons with this orientation, thus causing a longer lifetime for unpaired $2 s$ electrons with spins along the external field direction. The precession is then given by

$\mathrm{d} \phi=\frac{g \mu_{\mathrm{N}} B_{2 s} F(t) \zeta}{\hbar} \mathrm{d} t$

where $F(t)$ is the fraction of moving ions with one hole in the $2 \mathrm{~s}$ shell, $\zeta$ is the fraction of polarized $\mathrm{M}$ electrons in $\mathrm{Fe}, \mu_{\mathrm{N}}$ is the nuclear magneton and $B_{2 \mathrm{~s}}$ is the magnetic field at the nucleus caused by an unpaired $2 \mathrm{~s}$ electron. Non-zero values of $F(t)$ are only obtained when the cross sections for capture $\left(\sigma_{C}\right)$ and loss $\left(\sigma_{L}\right)$ of electrons are comparable in magnitude. It is easy to derive the expression

$F(t)=\frac{2 \sigma_{L}(t) \sigma_{C}(t)}{\left\{\sigma_{L}(t)+\sigma_{C}(t)\right\}^{2}}$.

The electron-loss cross section for the L-shell taken from Garcia et al. [14], which is of the order of $10^{9} \mathrm{~b}$, is given in fig. 2 . The dominant process in electron capture into the $2 \mathrm{~s}$ shell is non-radiative capture

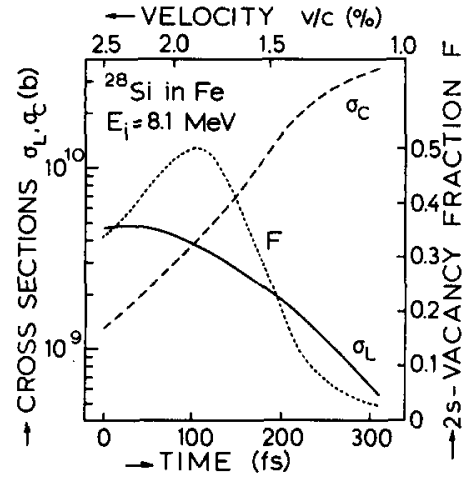

Fig. 2. Electron capture $\left(\sigma_{C}\right)$ and loss $\left(\sigma_{L}\right)$ cross sections as a function of flight time and velocity for an initial recoil energy $E_{\mathrm{i}}=8.1 \mathrm{MeV}$. The resulting single-2s-vacancy fraction $F$ is also shown.

of continuum electrons (Coulomb charge transfer). The cross section for radiative electron capture of continuum electrons is only of the order of $10^{2} b$ [15]. Radiative decay of bound electrons and Coster-Kronig transitions are improbable. The cross section $\sigma_{\mathrm{C}}(t)$ (see fig. 2) was estimated in the velocity region of interest from eq. (4.18) in ref. [15]. Velocity mismatch excludes transfer of $\mathrm{Fe} \mathrm{K}$ - and L-electrons to the $\mathrm{Si}$ ion. The fraction $\zeta=0.13$ of the M-electrons is polarized. Time integration of $F$ weighted with the decay of the nuclear state yields an average rotation of 2.4 mrad for the high-velocity point. The probability of $\mathrm{K}$-shell excitation [14] is less than $0.02 \%$ and may be neglected. Inclusion of $(1 s)^{2}(2 s)^{2}(2 p)^{-1} ;{ }^{2} P_{1 / 2}$ and $(1 s)^{2}(2 s)^{2}(2 p)^{6}(3 s)^{-1} ;{ }^{2} S_{1 / 2}$ configurations lead to an estimated total precession of $3.0 \mathrm{mrad}$. This must be added to the $\mathrm{LW}$ precession.

The same analysis for the intermediate-velocity point leads to an extra precession of $0.8 \mathrm{mrad}$. Both values give good quantitative agreement with the observed precessions. The sum of the precessions caused by polarized electron scattering ( $L W$ theory) and by polarized electron capture (PEC) is given by the dotted curve in fig. 1.

For the lighter ions ${ }^{16} \mathrm{~N}$ and ${ }^{18} \mathrm{O}[8,9]$ polarized electron capture in the K-shell becomes more likely and can account for the effects observed. The measured effects in heavier nuclei may be accounted for by polarized electron capture in higher-s orbits (4s-6s).

The observed effects may enable the measurement of $\boldsymbol{g}$-factors of extremely short-lived states. 
Stimulating discussions with Dr. F.W. Saris are gratefully acknowledged. This work was performed as part of the research program of the "Stichting voor Fundamenteel Onderzoek der Materie" (F.O.M.) with financial support from the "Nederlandse Organisatie voor Zuiver-Wetenschappelijk Onderzoek” (Z.W.O.).

\section{References}

[1] G.K. Hubler, H.W. Kugel and D.E. Murnick, Phys. Rev. C9 (1974) 1954.

[2] J.L. Eberhardt, R.E. Horstman, H.W. Heeman and G. Van Middelkoop, Nucl. Phys. A229 (1974) 162.

[3] J.L. Eberhardt, R.E. Horstman, H.A. Doubt and G. Van Middelkoop, Nucl. Phys., to be published.
[4] J. Lindhard and A. Winther, Nucl. Phys. A166 (1971) 413.

[5] G.M. Heestand, P. Hvelplund, B. Skaali and B. Herskind, Phys. Rev. B2 (1970) 3698.

[6] R. Heusinger et al., Internal report Erlangen-Nürnberg, Germany.

[7] G.K. Hubler, Ph.D. thesis, Rutgers University (1972).

[8] M. Forterre et al., Phys. Lett. 55B (1975) 59; Phys. Rev. $\mathrm{C}$, to be published.

[9] J.F.A. van Hienen (Utrecht University), private communication.

[10] J.F.A. van Hienen, P.W.M. Glaudemans and J. van Lith de Jeude, Nucl. Phys. A225 (1974) 119.

[11] M.R. Gunye, Phys. Lett. 37B (1971) 125.

[12] J.E. Koops (Utrecht University), private communication.

[13] R.E. Horstman et al., Nucl. Phys., to be published.

[14] J.D. Garcia, R.J. Fortner and T.M. Kavanagh, Revs. Mod. Phys. 45 (1973) 111.

[15] H.D. Betz, Revs. Mod. Phys. 44 (1972) 465. 